\title{
EQUICONTINUITY AND ALMOST PERIODIC FUNCTIONS ${ }^{1}$
}

\section{ROBERT ELLIS}

Let $X$ be a separated uniform space, $C(X, X)$ the set of continuous functions of $X$ into $X$, and $C(X)$ the set of real valued continuous functions on $X$ provided with the topology of uniform convergence. For $f \in C(X)$ and $a \in C(X, X), f a$ will denote that element of $C(X)$ such that $(f a)(x)=f(x a)(x \in X)$. Let $f \in C(X)$ and $A \subset C(X, X)$. Then $f$ is said to be almost periodic with respect to $A$ if $f A=[f a \mid a \in A]$ is a relatively compact subset of $C(X)$.

In this paper the behavior of $A$ is studied by means of its almost period functions. In particular it is shown that if there are enough of these, then $A$ is equicontinuous. This result is used to extend a result of Baum's [1] to nonabelian groups (Theorem 1), and to provide another proof of Maak's result that every right almost periodic function in the sense of von Neumann is also left almost periodic and vice-versa (Theorem 2). In Theorem 3 the effect of weakening the definition of almost periodic functions is studied, and in Theorem 4 the local behavior of a group of homeomorphisms is studied by means of its almost periodic functions.

Lemma 1. Let $A \subset C(X, X)$, let $Q$ be the set of almost periodic functions with respect to $A$, and let $U_{Q}$ be the uniform structure determined by $a$, (i.e. the smallest uniformity making each element of a uniformly continuous). Then

(1) $A$ is a set of equicontinuous maps of $X$ into $\left(X, U_{Q}\right)$.

(2) If fa $\in Q$ for all $f \in Q$ and $a \in A$, then $A$ is a set of uniformly equicontinuous maps of $\left(X, U_{Q}\right)$ into $\left(X, U_{Q}\right)$.

(3) If $X$ is compact, then $A$ is equicontinuous if and only if a separates points. In this case $\mathrm{Q}=C(X)$.

Proof. (1) A subbase of $U_{Q}$ is given by all the sets of the form $[f ; \epsilon]=[(x, y) \mid x, y \in X$ and $|f(x)-f(y)|<\epsilon]$ where $f \in Q$ and $\epsilon>0$. By assumption $f A$ is a relatively compact subset of $C(X)$. Since $C(X)$ is complete, $f A$ is totally bounded; i.e. there exists a finite

Presented to the Society, January 22, 1959; received by the editors February 12, 1959.

1 This research was supported by the United States Air Force through the Air Force Office of Scientific Research of the Air Research and Development Command, under contract AF18 (600)-116. Reproduction in whole or in part is permitted for any purpose of the United States Government. 
subset $B$ of $A$ such that $f A \subset f B(X, \epsilon / 3)$, where $f B(X, \epsilon / 3)$ $=[\psi \mid \psi \in C(X)$ and there exists $b \in B$ with $|\psi(x)-(f b)(x)|<\epsilon / 3$ $(x \in X)]$. Let $x \in X$. Then there exists a neighborhood $V$ of $x$ such that $|(f b)(x)-(f b)(y)|<\epsilon / 3 \quad(y \in V, b \in B)$. Let $a \in A$. Then there exists $b \in B$ such that $|f(z b)-f(z a)|<\epsilon / 3 \quad(z \in X)$. Thus if $y \in V$ $|f(x b)-f(y b)|<\epsilon / 3, \quad|f(x a)-f(x b)|<\epsilon / 3$, and $|f(y a)-f(y b)|<\epsilon / 3$; whence $|f(x a)-f(y a)|<\epsilon$. Hence $y \in V$ implies that $(x a, y a) \in[f ; \epsilon]$ for all $a \in A$. The proof is completed.

(2) Let $\epsilon>0, f \in a$, and $B$ a finite subset of $A$ such that $f A \subset f B(X, \epsilon / 2)$. Then a direct computation shows that if $(x, y)$ $\in[f B ; \epsilon / 2]$ than $(x a, y a) \in[f ; \epsilon]$. Since $[f B ; \epsilon / 2] \in U_{Q}$ by assumption, the proof is completed.

(3) Let $X$ be compact and suppose $a$ separates points. Then $U_{a}$ is a separated uniformity and hence coincides with the original uniformity on $X$. Consequently $A$ is equicontinuous by (1).

Conversely suppose $X$ compact and $A$ equicontinuous. Then by Ascoli's Theorem $A$ is a relatively compact subset of $C(X, X)$ when this set is provided with the topology of uniform convergence. Hence $f A$ is a relatively compact subset of $C(X)(f \in C(X))$, because the map $a \rightarrow f a(a \in C(X, X))$ of $C(X, X)$ into $C(X)$ is continuous for all $f \in C(X)$.

The characterization of equicontinuity given in (3) of Lemma 2 gives the following:

Corollary 1. Let $X$ be compact and let $A \subset C(X, X)$. Then $A$ is equicontinuous if and only if every countable subset of $A$ is equicontinuous.

Proof. Assume every countable subset of $A$ is equicontinuous, and let $f \in C(X)$. Then by Lemma 1 all that needs to be shown is that $f A$ is a relatively compact subset of $C(X)$. Since $C(X)$ is metrizable, this reduces to showing that $f B$ is a relatively compact subset of $C(X)$ for all countable subsets $B$ of $A$. But this latter condition follows from the assumption and Lemma 1.

Let $(X, G, \pi)$ be a transformation group [5]. A subset $A$ of $G$ is said to be syndetic if there is a compact subset $K$ of $G$ such that $K A=G$.

Lemma 2. Let $(X, G, \pi)$ be a transformation group with compact phase space $X$, and let $f \in C(X)$. Then $f$ is almost periodic with respect to $G$ if and only if for every $\epsilon>0$ there exists a syndetic subset $A$ of $G$ such that $|f(x a)-f(x)|<\epsilon(x \in X, a \in A)$.

Proof. Let $\epsilon>0, f \in C(X)$ and suppose $f$ is almost periodic with 
respect to $G$. Then $f G$ is a totally bounded subset of $C(X)$. Hence there exists a finite subset $F$ of $G$ such that $f G \subset f F(X, \epsilon)$. For $a \in F$ set $A_{a}=[g \mid g \in G$ and $f g \in f a(X, \epsilon)]$. Let $A=\bigcup\left[a^{-1} A_{a} \mid a \in F\right]$. Then $F A=G$ and $|f(x a)-f(x)|<\epsilon(x \in X, a \in A)$.

Now suppose $f$ satisfies the condition expressed in the lemma. Let $\epsilon>0$. Then there is a syndetic subset $A$ of $G$ such that $|f(x a)-f(x)|$ $<\epsilon / 2(x \in X, a \in A)$. Let $K$ be a compact subset of $G$ such that $K A=G$. Then another way of expressing the above relationship is that $f G \subset f K(X, \epsilon / 2)$. Since $K$ is a compact subset of $G$ and $\pi$ is continuous, $K$ is a compact subset of $C(X, X)$ provided with the topology of uniform convergence. Hence $f K$ is a compact subset of $C(X)$. Thus there exists a finite subset $F$ of $K$ such that $f K \subset f F(X, \epsilon / 2)$. Then $f G \subset f F(X, \epsilon)$. The proof is completed.

Let $f \in C(X), x \in X$, and $B \subset C(X, X)$. Then $f_{x}$ denotes that element of $C(B)$ such that $f_{x}(b)=f(x b)(b \in B)$. If $a \in C(X, X)$ then $f_{x} a$ is that element of $C(B)$ such that $\left(f_{x} a\right)(b)=f_{x}(b a)=f(x b a)(b \in B)$.

Lemma 3. Let $X$ be compact, $A \subset C(X, X)$, and for each $f \in C(X)$ let there exist $x \in X$ and $B \subset C(X, X)$ such that $f_{x} A$ is a relatively compact subset of $C(B)$ and such that $x B=[x b \mid b \in B]$ is an everywhere dense subset of $X$. Then $A$ is equicontinuous.

Proof. Let $f \in C(X)$. Then there exist $x \in X$ and $B C C(X, X)$ with the properties specified in the theorem. Now let $\mathcal{F}$ be an ultrafilter on $A$ and let $\epsilon>0$. Since $f_{x} A$ is a relatively compact subset of $C(B)$, $f_{x} \mathfrak{F}$ converges uniformly to some element of $C(B)$. Hence there is $F \in \mathcal{F}$ such that if $a_{1}, a_{2} \in F$, then $\left|\left(f_{x} a_{1}\right)(b)-\left(f_{x} a_{2}\right)(b)\right|<\epsilon(b \in B)$. In other words $\left|f\left(x b a_{1}\right)-f\left(x b a_{2}\right)\right|<\epsilon\left(b \in B, a_{1}, a_{2} \in F\right)$. Since $(x B)^{-}=X$, this implies that $\left|f\left(y a_{1}\right)-f\left(y a_{2}\right)\right|<\epsilon\left(y \in X, a_{1}, a_{2} \in F\right)$. Consequently $f F$ is a cauchy filter on $C(X)$ and so converges. Then $f A$ is a relatively compact subset of $C(X)$. Lemma 3 now follows from (3) of Lemma 1.

The following generalization of Baum's [1] result now follows from Lemmas 2 and 3. For the definition of the terms involved see the reference cited above.

Theorem 1. Let $(X, G, \pi)$ be a transformation group with compact phase space $X$ such that for each $f \in C(X)$ there exists $x \in X$ with $(x G)^{-}=x$ such that $f_{x}$ is almost periodic in the right transformation group over $G$ to the reals $R$. Then $G$ is equicontinuous.

Let $S$ be a semigroup, $s \in S$, and $f \in C(S)$. Then $f^{s}$ and $f_{s}$ will denote those elements of $C(S)$ such that $f^{s}(t)=f(s t)(t \in S)$ and $f_{s}(t)$ $=f(t s)(t \in S)$. Also $f^{S}$ and $f_{S}$ will denote the sets $\left[f^{s} \mid s \in S\right]$ and $\left[f_{s} \mid s \in S\right]$ respectively. 
THEOREM 2. Let $S$ be a semigroup with identity $e$ and let $f \in C(S)$. Then $f^{S}$ is a relatively compact subset of $C(S)$ if and only if $f_{S}$ is one also.

Proof. Let $f \in C(S)$ and assume $f^{S}$ is a relatively compact subset of $C(S)$. The map $\psi \rightarrow \psi(e)(\psi \in C(S))$ of $C(S)$ into the reals $R$ is continuous. Hence $f(S)$ which is the image of $f^{S}$ under this map is a relatively compact subset of $R$. Let $J=\left(f_{S}\right)^{-}$. Then $f_{S} \subset J^{S}$. Let $X$ be the closure of $f_{S}$ in this space. For $x \in X$ and $s \in S$ let $x s$ denote that element of $J^{s}$ such that $(x s)(t)=x(s t) \quad(t \in S)$. Then the mapping $x \rightarrow x s(x \in X)$ of $X$ into $J^{S}$ is continuous for all $s \in S$. Moreover if $r, s \in S$, then $\left(f_{r} s\right)(t)=f_{r}(s t)=f(r s t)=f_{r s}(t)$; i.e. $f_{r} s=f_{r s}$ and so $f_{S} \subset \subset f_{S}$ $\subset X$. Hence $x s \in X(x \in X, s \in S)$.

The aim now is to show that $S$ is a equicontinuous collection of maps of $X$ into $X$. To this end let $a \in S$ and let $\psi_{a}$ denote that element of $C(X)$ such that $\psi_{a}(x)=x(a)(x \in X)$. Recall that in conformity with the notation used previously if $s \in S$ then $\psi_{a} s$ denotes that element of $C(X)$ such that $\left(\psi_{a} s\right)(x)=\psi_{a}(x s) \quad(x \in X)$. Thus $\left(\psi_{a} s\right)(x)$ $=(x s)(a)=x(s a)(s \in S, x \in X)$. Now let $\mathcal{F}$ be an ultrafilter on $S$. Then $\mathcal{F} a=[F a \mid F \in \mathcal{F}]$ is an ultrafilter base on $S$ and so by assumption $f F^{a}=\left[f^{F a} \mid F \in \mathcal{F}\right]$ is a cauchy filter base on $C(S)$. Therefore, given $\epsilon>0$ there exists $F \in \mathcal{F}$ such that $|f(s b a)-f(s c a)|<\epsilon(s \in S, b, c \in F)$. In other words $\left|\left(\psi_{a} b\right)\left(f_{s}\right)-\left(\psi_{a} c\right)\left(f_{s}\right)\right|<\epsilon \quad(s \in S, b, c \in F)$. Since $\bar{f}_{S}=X$, this means that $\left|\left(\psi_{a} b\right)(x)-\left(\psi_{a} c\right)(x)\right|<\epsilon(x \in X, b, c \in F)$; i.e. $\psi_{a} F$ is a cauchy filter base on $C(X)$. Thus $\psi_{a}$ is an almost periodic function on $X$ with respect to $S$. By the definition of $X$ the set of functions $\left[\psi_{a} \mid a \in S\right]$ separates points of $X$. Hence $S$ is an equicontinuous family of maps of $X$ into $X$. Let $T$ be the closure of $S$ in $X^{X}$ and $\pi$ the map of $X \times T$ into $R$ such that

$$
\pi(x, t)=(x t)(e)(x \in X, t \in T) .
$$

Then the equicontinuity of $S$ implies the continuity of $\pi$. Therefore $X$ is a compact subset of $C(T)$ and a fortiori $f_{S}$ is a relatively compact subset of $C(S)$. The proof is completed.

Let $C_{0}(X)$ denote the set of bounded real valued continuous functions on $X$, let $A \subset C(X, X)$, and let $f \in C_{0}(X)$. Then following Eberlein [2] $f$ will be called weakly almost periodic with respect to $A$ if $f A$ is a relatively compact subset of $C_{0}(X)$ provided with the weak $\mathrm{Ba}$ nach space topology. The question now is what can be said about $A$ if every element of $C_{0}(X)$ is weakly almost periodic with respect to $A$ ? The following example shows that even when $X$ is compact and $A$ is a group, we cannot conclude that $A$ is equicontinuous. Let $G$ be 
a locally compact, noncompact topological group, and let $X$ be the one point compactification of $X$ with point at infinity, $z$. Then $G$ may be identified with the set of maps $x \rightarrow x g(x \in X, x \neq z)$ and $z g=z$ of $X$ into $X ;(g \in C)$. Then every element of $C(X)$ is weakly almost periodic with respect to $G$ but $G$ does not act equicontinuously on $X$.

Lemma 4. Let $X$ be compact and let $A \subset C(X, X)$. Then every element of $C_{0}(X)=C(X)$ is weakly almost periodic with respect to $A$ if and only if $A$ is a relatively compact subset of $C(X, X)$ provided with the topology of pointwise convergence.

Proof. Suppose every element of $C(X)$ weakly almost periodic with respect to $A$ and let $\mathcal{F}$ be an ultrafilter on $A$. Then $\mathcal{F}$ converges pointwise to an element $b$ of $X^{X}$. For $f \in C(X) f \mathcal{F}$ converges pointwise to $f b$. Hence $f b$ is a continuous mapping of $X$ into the reals. Consequently $b \in C(X, X)$.

For each $f \in C(X)$ the map $a \rightarrow f a$ of $C(X, X)$ into $C(X)$ is continuous when these sets are provided with the topologies of pointwise convergence. Thus if $A$ is a relatively compact subset of $C(X, X)$, then $f A$ is relatively compact when $C(X)$ is provided with the topology of pointwise convergence. Since $X$ is compact, $f A$ is relatively weakly compact [6]. The proof is completed.

Let $A \subset C(X, X)$. Then $A$ will be called distal if given any three points $x, y, z \in X$ and any filter $\mathcal{F}$ on $A$ such that $x \mathfrak{F} \rightarrow z$ and $y \mathcal{F} \rightarrow z$, then $x=y[5]$.

Theorem 3. Let $X$ be compact and let $S$ be a distal semigroup of elements of $C(X, X)$ such that every element of $C(X)$ is weakly almost periodic with respect to $S$. Then $S$ is equicontinuous and every element of $S$ is a homeomorphism onto.

Proof. Let $T$ be the closure of $S$ in $C(X, X)$ provided with the topology of pointwise convergence. Then $T$ is compact by Lemma 4 . Since $S^{2} \subset S, \bar{S}=T$, and the maps $t \rightarrow r t$ and $t \rightarrow t r(t \in C(X, X))$ of $C(X, X)$ into $C(X, X)$ are continuous for all $r \in C(X, X), T^{2} \subset T$.

Now let $t \in T$ and set $\varepsilon=[E / \varnothing \neq E \subset T t, E$ is compact, and $T E \subset E]$. Then $\varepsilon \neq \varnothing$ since $T t \in \mathcal{E}$. If $\mathcal{E}$ is ordered by inclusion then one verifies that Zorn's Lemma is applicable so that $\mathcal{E}$ has a minimal element $E$. Let $r \in E$. Then $\operatorname{Er} \subset E E \subset T E \subset E$, and $T E r \subset E r$. Thus $E r \in \mathcal{E}$ and $E r \subset E$. Therefore $E r=E$. Hence there exists $p \in E$ such that $p r=r$. Let $x \in X$ and $\mathfrak{F}$ a filter on $S$ such that $\mathfrak{F}$ converges pointwise to $r$. Let $x r=z$. Then $x p \mathcal{F}$ and $x \mathcal{F}$ both converge to $z$. Since $S$ is assumed to be distal, this means that $x p=x(x \in X)$. Hence $p=e$, the identity map of $X$. Then $e \in E$ and $T E \subset E$ implies that $E=T$. Moreover 
$E \subset T t$ implies that $T t=T$. Therefore, there exists $s \in T$ with $s t=e$. Similarly there exists $q \in T$ with $q s=e$; whence $q=q e=q s t=e t=t$; i.e. $s t=t s=e$. Thus $T$ is a group and so every element $S$ is a homeomorphism onto. The equicontinuity of $T$ and hence of $S$ now follows from [3]. The proof is completed.

The next theorem is concerned with the local behavior of a group of homeomorphisms. Let $x \in X$ and $a \subset C(X)$. Then $Q$ separates $x$ if for every $y \in X$ such that $y \neq x$ there exists $f \in Q$ with $f(x) \neq f(y)$. A transformation group $(X, G, \pi)$ is said to be locally almost periodic at $x \in X$ if given any neighborhood $U$ of $x$ there exist a neighborhood $V$ of $x$ and a syndetic subset $A$ of $G$ such that $V A=\pi(V \times A) \subset U$.

THEOREM 4. Let $(X, G, \pi)$ be a transformation group with compact phase space $X$ and let $x_{0} \in X$ be such that the almost periodic functions with respect to $G$ separate it. Then $G$ is locally almost periodic at $x_{0}$.

Proof. Let $a$ be the set of almost periodic functions with respect to $G$. For $x, y \in X$ set $x \sim y$ if and only if $f(x)=f(y)(f \in Q)$. Then $\sim$ is an equivalence relation on $X$. Let $\tilde{x}(x \in X)$ be the equivalence class to which $x$ belongs, $Y$ the quotient space, and $p$ the projection of $X$ onto $Y$.

For $x, y \in X, x \sim y$ implies that $x g \sim y g(g \in G)$, because $f g \in Q(f \in Q, g \in G)$. Thus $G$ may be regarded as a group of homeomorphisms of $Y$ onto $Y$; where $\tilde{x} g=(x g)^{\sim}(x \in X, g \in G)$. Also each $f \in Q$ may be identified with that element of $C(Y)$ which maps $\tilde{x}$ into $f(x)(x \in X)$.

For $x \in X, \tilde{x}$ may be identified with the map $f \rightarrow f(x)(f \in a)$ of $a$ into $R$. Hence $R^{\mathbb{Q}}$ induces a Hausdorff topology on $Y$. Since the maps $\tilde{x} \rightarrow f(x)(x \in X)$ of $Y$ into $R$ are continuous $(f \in Q)$, the quotient space topology on $Y$ contains the topology induced on $Y$ by $R^{Q}$. The compactness of the former implies that the two topologies coincide. Thus $Y$ is compact Hausdorff.

Let $f \in Q$ and $\mathcal{F}$ an ultrafilter on $G$. Then by assumption $f \mathcal{F}$ converges uniformly to $\psi \in C(X)$. This implies that if $x \sim y$ then $\psi(x)$ $=\psi(y)$ and so $\psi$ induces an element of $C(Y)$ to which $f \mathcal{F}$ converges uniformly on $Y$. Consequently $f$ is an almost periodic function on $Y$ with respect to $G$ for all $f \in a$. By the definition of $\sim$, $Q$ separates points of $Y$. Therefore $G$ is an equicontinuous family of maps of $Y$ into $Y$ by Lemma 1.

Now let $U$ be an open neighborhood of $x_{0}$ in $X$, and suppose $p^{-1}(W) \cap U^{\prime} \neq \varnothing$ for all neighborhoods $W$ of $\tilde{x}_{0}$ in $Y$, where $U^{\prime}$ is the complement of $U$. Since $p^{-1}\left(W_{1} \cap W_{2}\right) \cap U^{\prime} \subset p^{-1}\left(W_{1}\right) \cap U^{\prime} \cap p^{-1}\left(W_{2}\right)$ $\cap U^{\prime}$, this implies that $\left[p^{-1}(W) \cap U^{\prime} / W\right.$ closed neighborhood of $\left.\tilde{x}_{0}\right]$ 
is a family of compact subsets of $X$ having the finite intersection property. Thus there would be $x \in X$ with $x \in \cap\left[p^{-1}(W) / W\right.$ closed neighborhood of $\left.\tilde{x}_{0}\right] \cap U^{\prime}$; i.e. $x \neq x_{0}$ and $p(x)=\tilde{x}_{0}$. This contradicts the assumption on $x_{0}$ and the definition of $\sim$. Hence there exists a neighborhood $W$ of $\tilde{x}_{0}$ such that $p^{-1}(W) \subset U$.

Since $G$ is equicontinuous on $Y$, it is also locally almost periodic at $\tilde{x}_{0}$. Therefore there exist a neighborhood $N$ of $\tilde{x}_{0}$ and a syndetic subset of $A$ of $G$ such that $N A \subset W$. Set $V=p^{-1}(N)$. Then $V A \subset p^{-1}(W) \subset U$. The proof is completed.

THeOREM 5. Let $(X, G, \pi)$ be a transformation group with compact phase space $X$, let $G$ be distal, and let $x_{0} \in X$ be such that the almost periodic functions on $X$ with respect to $G$ separate it. Then $G$ is equicontinuous at $x_{0}$. If furthermore $\left(x_{0} G\right)^{-}=X$, then $G$ is equicontinuous.

Proof. The first part of the theorem follows immediately from Theorem 4 and Theorem 1 of [4]. The second part follows from the fact that if $\left(x_{0} G\right)^{-}=X$ and $G$ is locally almost periodic at $x_{0}$ then $G$ is locally almost periodic at all $x \in X,[5,4.11]$.

\section{REFERENCES}

1. John D. Baum, An equicontinuity condition for transformation groups, Proc. Amer. Soc. vol. 4 (1953) pp. 656-662.

2. F. Eberlein, Abstract ergodic theorems and weak almost periodic functions, Trans. Amer. Math. Soc. vol. 67 (1949) pp. 217-240.

3. Robert Ellis, Locally compact transformation groups, Duke Math. J. vol. 24 (1957) pp. 119-126.

4. W. H. Gottschalk, Characterizations of almost periodic transformation groups, Proc. Amer. Math. Soc. vol. 7 (1956) pp. 709-712.

5. W. H. Gottschalk and G. A. Hedlund, Topological dynamics, Amer. Math. Soc. Colloquium Publications, vol. 36, 1955.

6. A. Grothendieck, Criteres de compacite dans les espaces fonctionnels generaux, Amer. J. Math. vol. 74 (1952) pp. 168-186.

7. W. Maak, Eine neue Definition der fastperiodischen Funktionen, Abh. Math. Sem. Univ. Hamburg vol. 11 (1936) pp. 240-244.

University of Pennsylvania 